\title{
Reduciendo la incertidumbre en la ejecución de un piloto de combustión in situ en un campo de crudo extra pesado colombiano mediante la realización de una prueba de conectividad con nitrógeno
}

\author{
M. Trujillo*; E. Rodriguez; C. Delgadillo; Y. Claro; R. Rojas; J. Sandoval \\ ${ }^{1}$ Instituto Colombiano del Petróleo (ICP). Vía Piedecuesta Km 7. Piedecuesta, Colombia \\ *E-mail: martha.trujillo@ecopetrol.com.co
}

\begin{abstract}
Resumen
Antes de comenzar cualquier proceso de recuperación mejorada de petróleo (EOR), es deseable caracterizar el patrón de flujo dentro del volumen del yacimiento afectado. Esto se vuelve de importancia crítica para la combustión in situ en yacimientos de petróleo pesado, donde la relación de movilidad es altamente desfavorable, a menudo dando como resultado una canalización o un avance temprano del frente de inyección o combustión. Una prueba de conectividad entre pozos a través de inyecciones de gas inmiscible contribuye a mejorar la caracterización de la distribución de flujo de gas, además de: 1) Estimar eficiencias de barrido, 2) evidenciar características geológicas que pueden conducir a un flujo preferencial hacia un pozo o grupo particular de ellos, o falta de conexión entre ellos, 3) creación de una ruta de gas entre el inyector y los pozos productores para permitir una progresión segura del frente de combustión, y 4) evaluación del rendimiento de los sistemas de levantamiento artificial y sistemas de control de pozos en condiciones de alta relación gas-líquido. Se diseñó, implementó y evaluó una prueba de conectividad usando nitrógeno en el campo Chichimene, antes del inicio del piloto de combustión in situ. Este proceso se resume y describe en este documento. Esta será la primera prueba de combustión in situ en un yacimiento de petróleo extra-pesado profundo ( $\approx 8000$ pies $)$ a nivel mundial y servirá como una fuente de datos para evaluar el desarrollo de recursos en condiciones similares en la cuenca de los llanos orientales de Colombia. Este conjunto de yacimientos tiene una fracción significativa de los recursos de hidrocarburos en el país y están bajo la operación de Ecopetrol. La importancia de este piloto hace que esta prueba de conectividad tenga una relevancia aún mayor para reducir las incertidumbres de subsuelo y operativas, identificar riesgos y aumentar la probabilidad de éxito del proceso de combustión como una opción para producir estos recursos de manera económicamente rentable.
\end{abstract}

Palabras clave: Inyección de aire, crudo extra-pesado, prueba de conectividad, inyección de nitrógeno, recobro mejorado de petróleo.

\section{Reducing uncertainty at the Chichimene in situ combustion pilot by means of a nitrogen connectivity test}

\begin{abstract}
Prior to start any Enhanced Oil Recovery (EOR) process, it is desirable to characterize the flow pattern within the affected reservoir volume. This becomes of critical importance for in situ combustion in heavy oil reservoirs, where the mobility ratio is highly unfavorable, oftentimes resulting in channeling or early breakthrough. An inter-well connectivity test through immiscible gas injection aids improving the characterization of the flow distribution, in addition to: 1) calibrating estimates for sweep efficiency; 2) evidencing geological features that may lead to preferential flow towards a particular well or group of them, or lack of connection amongst them; 3) creating a gas path between the injector and producer wells to enable a safe progression of the combustion front; and 4) evaluating the performance of artificial lift and well control systems under high gas-liquid ratio conditions. A connectivity test using nitrogen was designed, implemented and evaluated at the Chichimene field, prior to the ignition of the in situ combustion pilot. This process is summarized and described in this paper. This will be the first in situ combustion trial in a deep $(\approx 8000 \mathrm{ft}$.), extra-heavy oil reservoir, and will serve as a data source to evaluate the development of

Cita: Trujillo, M., Rodriguez, E., Delgadillo, C., Claro, Y., Rojas, R. \& Sandoval, J. (2018). Reduciendo la incertidumbre en la ejecución de un piloto de combustión in situ en un campo de crudo extra pesado colombiano mediante la realización de una prueba de conectividad con nitrógeno. Revista Fuentes: El reventón energético, 16(2), 109-120.
\end{abstract}


resources under similar conditions in the eastern plains basin of Colombia. This set of reservoirs bears a significant fraction of the hydrocarbon resources in the country and under Ecopetrol operation. The importance of this pilot makes this connectivity test of even larger relevance to reduce the subsurface and operational uncertainty, identifying potential risks, and increase the probability of success of the combustion process as an option to economically produce these resources.

Keywords: Extra-heavy oil; Connectivity test; Nitrogen injection; Air injection; Enhanced Oil Recovery.

\section{Introducción}

La combustión in situ (CIS) es una técnica EOR que se basa en reacciones in situ entre el aceite del yacimiento y el aire comprimido inyectado (Sarathi, 1998; Navarro et al, 2016; Castañeda et al, 2014). La eficiencia del barrido se ve mejorada por una serie de mecanismos que implican la destilación y el craqueo en el frente de combustión, así como la condensación, el empuje del agua caliente y el desplazamiento inmiscible típicamente aguas abajo del mismo. En los aceites pesados, el frente de combustión se forma donde el oxígeno se consume rápidamente al reaccionar con un material similar al coque depositado después de una serie de reacciones de adición, craqueo y oxidación. Estas reacciones son de naturaleza exotérmica y producen altas temperaturas cerca del frente (Cañas, 2014). El calor puede ser transportado desde el frente por el agua en fase de vapor, que desempeña el papel principal en el desplazamiento, dejando el aceite residual que luego se transformará químicamente cuando el frente de oxidación alcanza esta posición (Burger, 1972; Fassihi, Brigham and Ramey, 1984).

La CIS se ha aplicado en yacimientos de petróleo pesado poco profundos y en yacimientos de petróleo liviano profundos (Turta and Singhal, 2001; Moore, Mehta and Ursenbach, 2002). Chichimene será la primera implementación de CIS en yacimientos de petróleo extra pesado y profundo. La formación San Fernando (también conocida como unidad T2) es el objetivo para el desarrollo con CIS. Está ubicado a una profundidad vertical que varía entre 7,700 y 8,300 pies, con un estimado de $2.76 \times 10^{9}$ STB de petróleo original en sitio (OIIP), y una temperatura inicial del yacimiento de $186^{\circ} \mathrm{F}$. La recuperación final estimada (EUR) por producción primaria ronda el $10 \%$. Aunque la alta temperatura del yacimiento favorece la reactividad del aceite in situ, la profundidad también presenta riesgos significativos asociados con una presión más alta, los costos de los pozos y la eficiencia de la compresión. El análisis inicial aplicado a Chichimene y yacimientos similares con una cantidad importante de reservas probadas, muestra que los procesos basados en vapor no son viables, dejando CIS como la única opción factible para EOR térmico y factores de recuperación altos (Aya, 2013; Martin, 2017).

Este proyecto piloto es de importancia estratégica para Ecopetrol, y la prueba de conectividad es una fuente sustancial de información que ayudará a reducir la incertidumbre de subsuelo para la fase de inyección de aire. Con este objetivo en mente, la prueba de conectividad de nitrógeno (PCN) se diseñó, implementó y evaluó como un esfuerzo interdisciplinario entre las operaciones, la administración técnica y los equipos de investigación y tecnología.

Al estudiar experiencias previas en el uso de trazadores para EOR, y más específicamente en CIS, particularmente antes de la etapa de inyección de aire (Johnson, 1980; Hutchinson, 1979; Pebdani, 1988 \& Glandt, 1998) se formularon los objetivos de la PCN y se definió un diseño conceptual.

En este artículo, se presentan los objetivos y las etapas de la PCN. Luego, se discute el diseño de la prueba basada en simulaciones numéricas. Más tarde, se informan los resultados de la PCN, para finalizar con el análisis y las conclusiones.

\section{Metodología}

\section{Objetivos y alcance de la prueba de conectividad}

Los objetivos establecidos para la PCN se enumeran en la Figura 1. Para cumplir con estos objetivos, se consideraron tres etapas: diseño, implementación y evaluación. En la etapa de diseño, se empleó un simulador de yacimiento comercial para estimar los rangos de operación esperados durante la prueba, mientras se considera la incertidumbre en una serie de parámetros de yacimiento. En la etapa de implementación, se siguieron las pautas de diseño y se reportaron las desviaciones y otras mediciones. Por último, en la etapa de evaluación, los datos reportados se utilizaron para generar gráficos y patrones que se analizaron para formular conclusiones y recomendaciones para la operación piloto. 


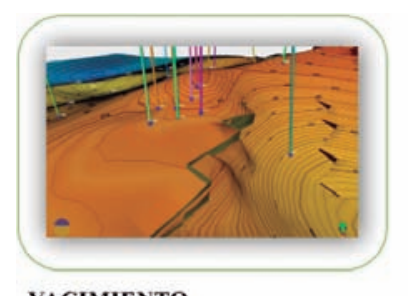

YACIMIENTO

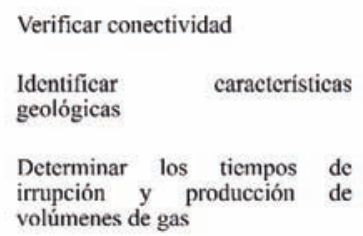

\section{OPERACIONAL}

Validar inyectividad y completamiento del inyector

Validar el desempeño del sistema de levantamiento artificial en pozos productores

Evaluar maniobras en pozos productores durante la etapa de homogeneizaciōn

Validar la estrategia de monitoreo y medición en el campo

\section{Etapas}

\section{Diseño de la prueba de conectividad}

La prueba piloto de combustión in situ en el campo Chichimene apunta a la formación T2, y está compuesta por un único pozo inyector (INJ), dos pozos observadores (OB1 y OB2) y tres pozos productores de primera línea (P3, P2 y P1), dentro de un área de aproximadamente 10 acres. Además, se espera que una segunda línea de productores tenga influencia de los gases de combustión, pero no de la zona de combustión a alta temperatura, y se incluyen en la estrategia de monitoreo. La ubicación de los pozos en el modelo sector se muestra en la Figura 2. Dentro de la formación T2, la unidad con las mejores propiedades petrofísicas es T2_40, por lo que todos los pozos piloto se completan dentro de esta unidad. Un registro tipo se presenta en la Figura 3.

Figura 1. Objetivos de la prueba de conectividad.

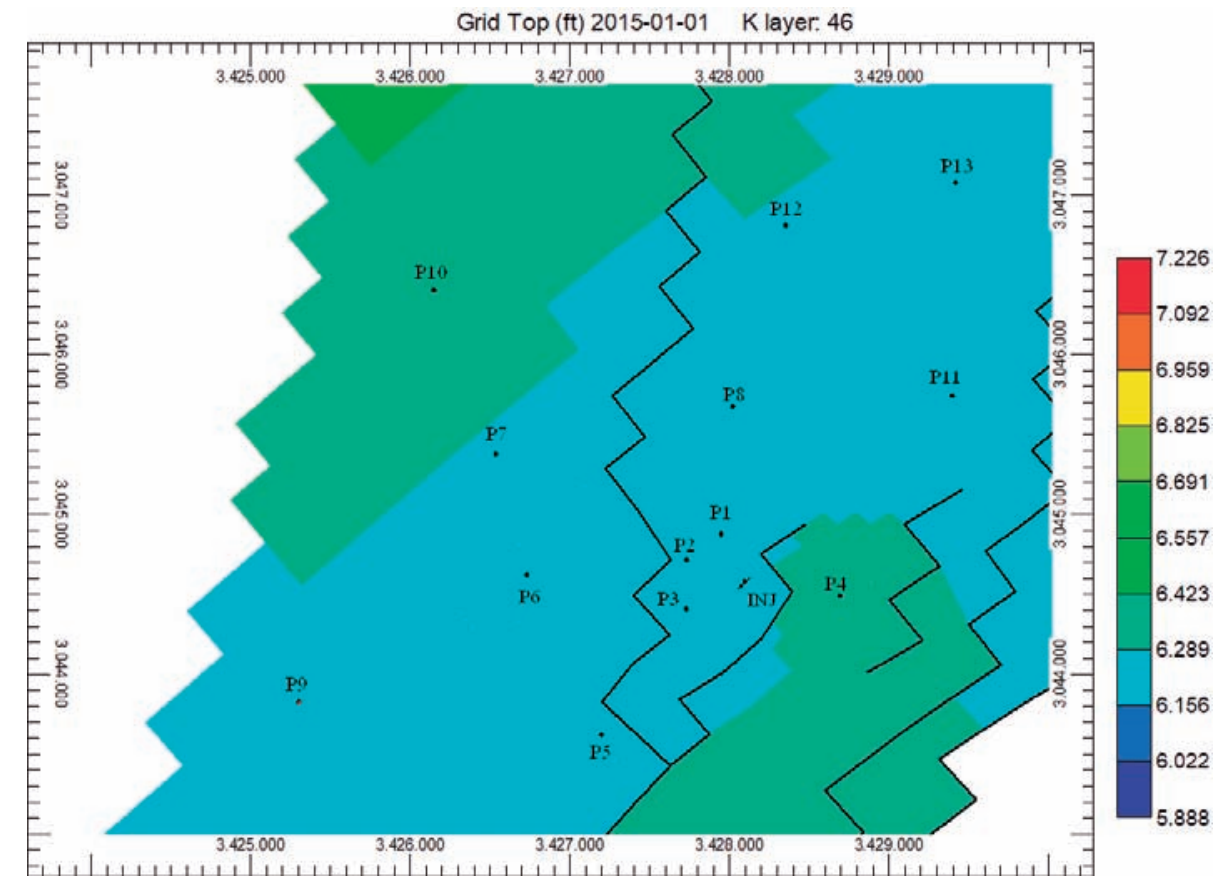

Figura 2. Posicionamiento de pozos para el piloto de combustión in situ.

Para evaluar una serie de escenarios probables durante la inyección de nitrógeno, se llevó a cabo la simulación numérica de yacimientos utilizando un software comercial. Se generó un modelo de simulación de alta resolución para el área piloto, prestando especial atención a la preservación de las características geológicas que influyen en la respuesta a la inyección de un fluido de alta movilidad en la formación. La Figura 4 representa la malla utilizada para las simulaciones, refinada localmente alrededor del inyector y los pozos productores de primera línea.

El ajuste histórico del comportamiento de producción primaria fue crucial para representar los datos de presión al comienzo de la inyección de nitrógeno. Las Figuras 5 a 7 ilustran el ajuste de la presión de fondo de pozo obtenido para cada uno de los pozos productores de primera línea. 


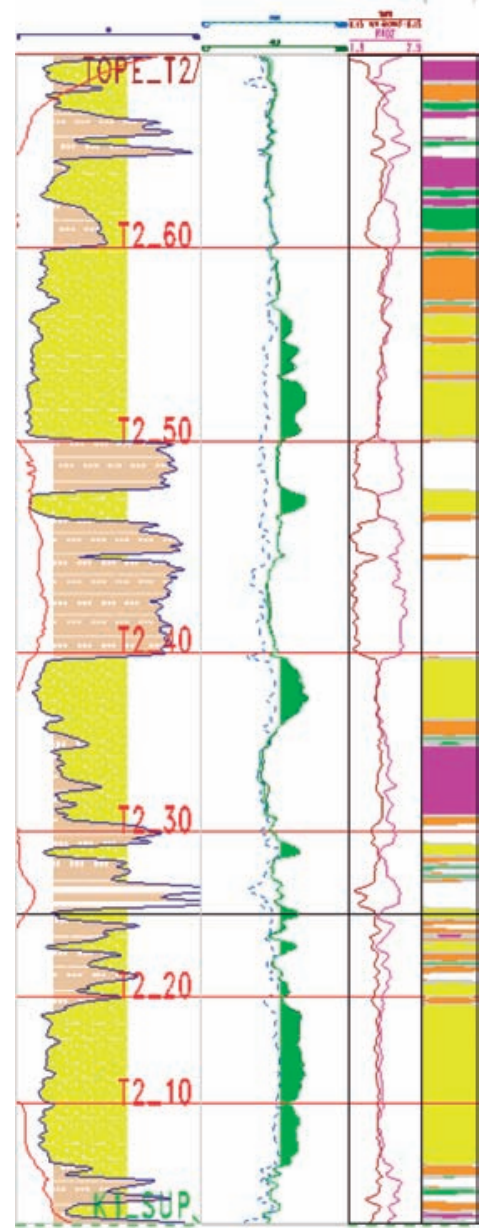

Figura 3. Sub-unidades de la formación T2. Líneas rojas representan topes.
Se eligió un conjunto de variables para evaluación mediante un análisis de sensibilidad: intervalo perforado y tasa de inyección que son parámetros que pueden establecerse, mientras que la relación de permeabilidad vertical a horizontal, los end points de permeabilidad relativa gas-líquido y la saturación de gas crítica son parámetros de yacimiento con un alto nivel de incertidumbre. Se determinó la respuesta a las tasas de producción de gas, las presiones de fondo del pozo (BHP) y los tiempos de avance. Las tablas de la 1 a la 3 resumen los casos estudiados para los parámetros críticos identificados.

La sensibilidad del tiempo de irrupción en función de la relación $\mathrm{kv} / \mathrm{kh}$ no fue significativa, por lo que el caso base se estableció en un valor de 0,6. Este parámetro mostró un ajuste histórico aceptable de la PCN. El escenario más favorable para el intervalo perforado se consideró como el Caso 2, lo que resulta en una mejor eficiencia de barrido vertical, como se evidencia en la Figura 8. De manera similar, para tener suficiente tiempo para observar el avance y determinar el cambio en la composición del gas se decidió el Caso 3 para el programa de inyección. También se decidió que, en caso de un tiempo de tránsito más largo, la tasa de inyección se incrementaría para promover el avance hacia los pozos de primera línea y acelerar la progresión del frente de nitrógeno hacia los pozos de segunda línea, mientras se mantiene un tiempo de prueba total razonable.

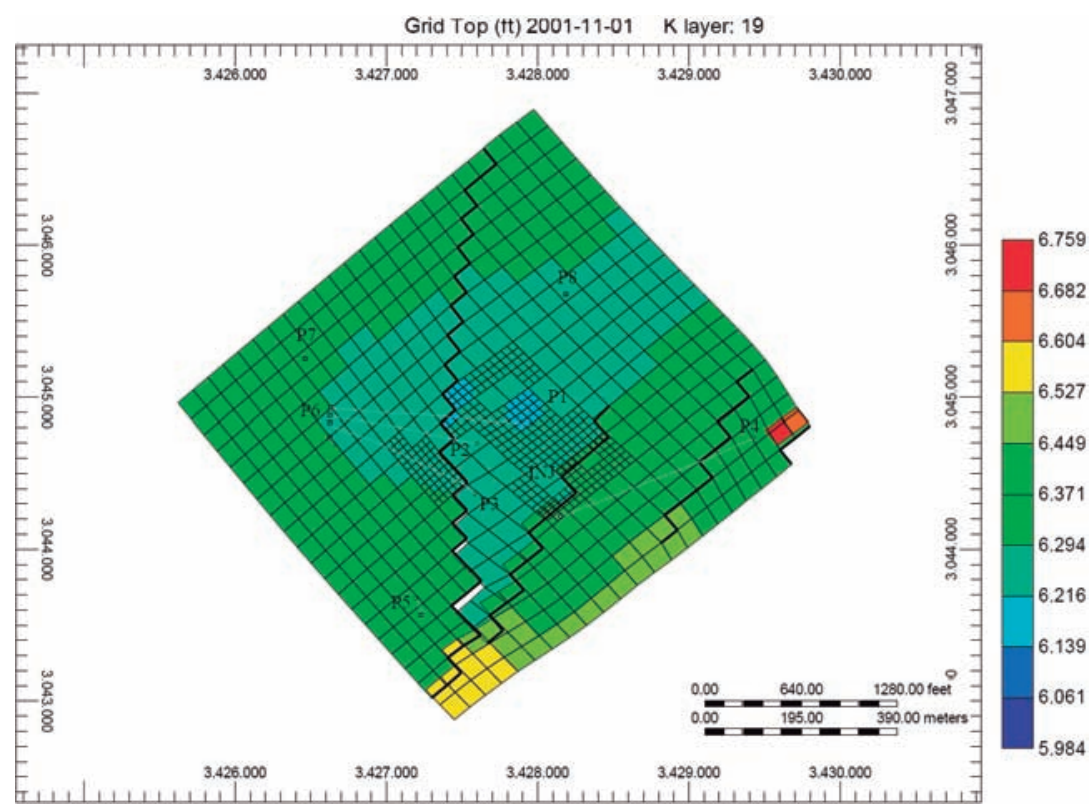

Figura 4. Modelo usado para las simulaciones de la PCN. 


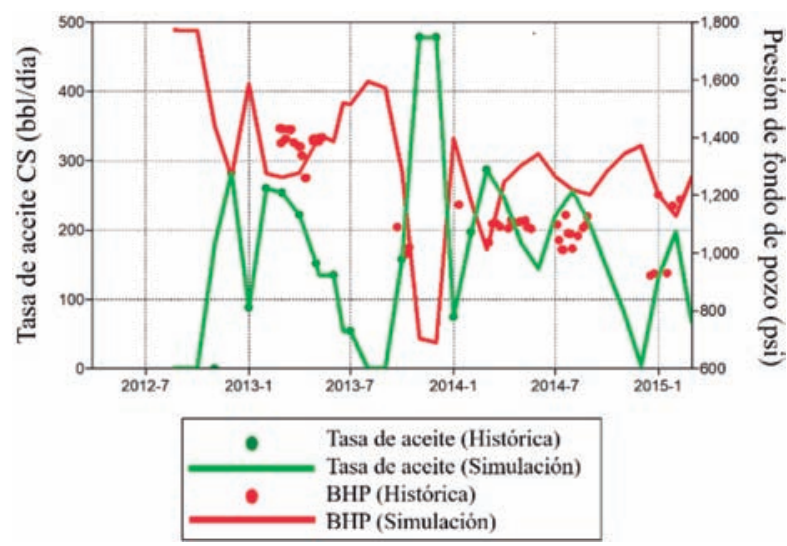

Figura 5. Ajuste Histórico del pozo P3.

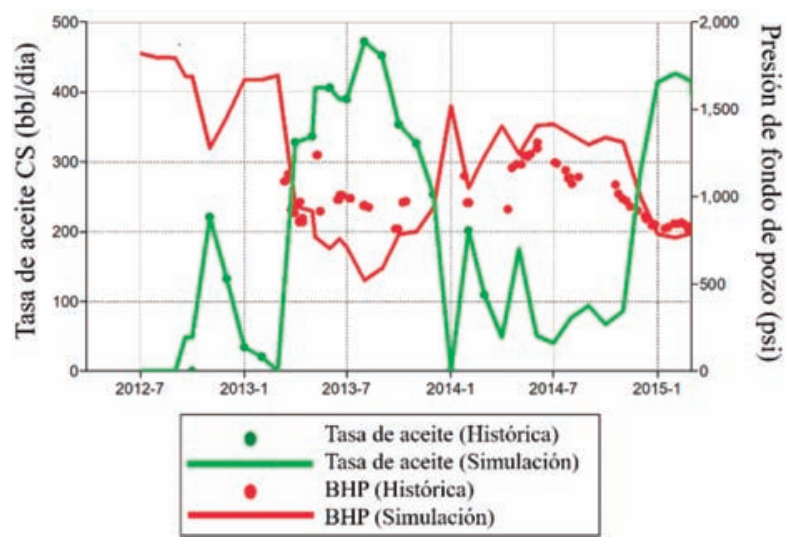

Figura 6. Ajuste histórico del pozo P2.

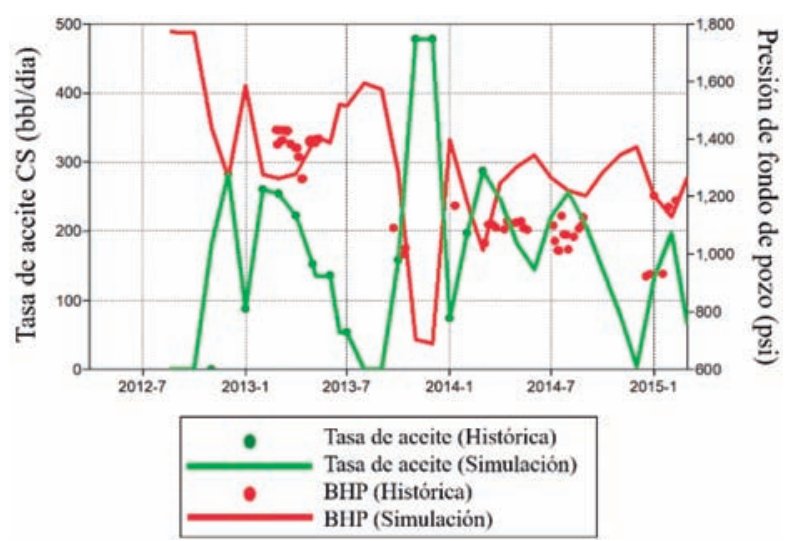

Figura 7. Ajuste histórico del pozo P1.

Tabla 1. Casos evaluados para el espesor perforado en el inyector.

\begin{tabular}{|c|c|c|}
\hline \multirow{2}{*}{ Caso } & \multicolumn{2}{|c|}{ Espesor perforado (ft) } \\
\cline { 2 - 3 } & Fondo T2_40 & T2_30 \\
\hline 1 & 20 & - \\
\hline 2 & 10 & 25 \\
\hline 3 & 20 & 25 \\
\hline
\end{tabular}

Tabla 2. Casos evaluados para la relación $\mathrm{Kv} / \mathrm{Kh}$.

\begin{tabular}{|c|c|}
\hline Caso & Relación $\mathbf{k}_{\mathbf{v}} / \mathbf{k}_{\mathbf{h}}$ \\
\hline 1 & 0,4 \\
\hline 2 & 0,6 \\
\hline 3 & 0,8 \\
\hline
\end{tabular}

Tabla 3. Casos evaluados para la tasa de inyección de nitrógeno.

\begin{tabular}{|c|c|}
\hline Caso & Tasa de Inyección (kSCF/d) \\
\hline 1 & 250 \\
\hline 2 & 250 (día 1), 500 (posteriormente) \\
\hline 3 & 250 (día 1), 500 (día 2), 750 (día 3), 1000 \\
(posteriormente)
\end{tabular}
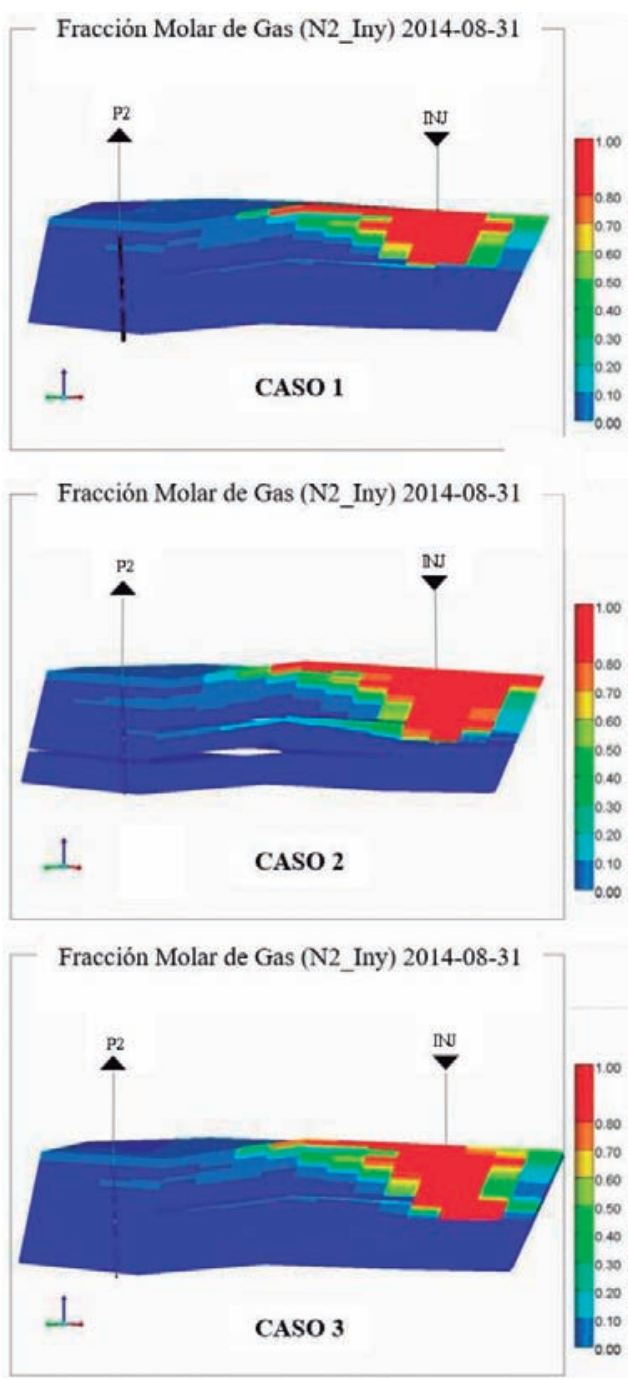

Figura 8. Efecto del intervalo perforado sobre la eficiencia de barrido vertical durante la PCN. 
Considerando la anisotropía en los datos de presión del campo creado por la diferencia observada en los datos de presión de fondo fluyendo de los pozos productores del piloto, se espera que el desplazamiento de nitrógeno hacia estos pozos no sea uniforme. La simulación numérica se usó para determinar la viabilidad de establecer una progresión más uniforme del frente de nitrógeno hacia los pozos productores mediante el ajuste de su flujo con la BHP durante la prueba. Esta situación óptima se caracterizaría por tiempos de irrupción del nitrógeno similares y tasas de producción de nitrógeno comparables. Esto se puede controlar en el simulador ajustando periódicamente el BHP después de considerar las discrepancias en la tasa de producción en ese período de tiempo. La Figura 9 muestra la realización correspondiente al programa de tasas de inyección seleccionadas con los intervalos perforados elegidos, donde se obtuvo una homogenización satisfactoria de la producción de nitrógeno a pesar de las discrepancias iniciales en BHP. Los tiempos de irrupción se consideraron más largos de lo esperado, solo para evidenciar que es posible una progresión homogénea del frente de nitrógeno durante un período de tiempo prolongado.

Dado el tiempo de irrupción esperado, la tasa de gas y BHP, se diseñó una estrategia de monitoreo. Esto involucra la frecuencia del muestreo de gas para capturar adecuadamente los tiempos de Irrupción, la calibración de los instrumentos de medición de gases y los ajustes a los dispositivos de control de pozos. El pozo inyector se completó de acuerdo con la recomendación del diseño, y el procedimiento operativo detallado para la implementación de la PCN también se basó en este trabajo.
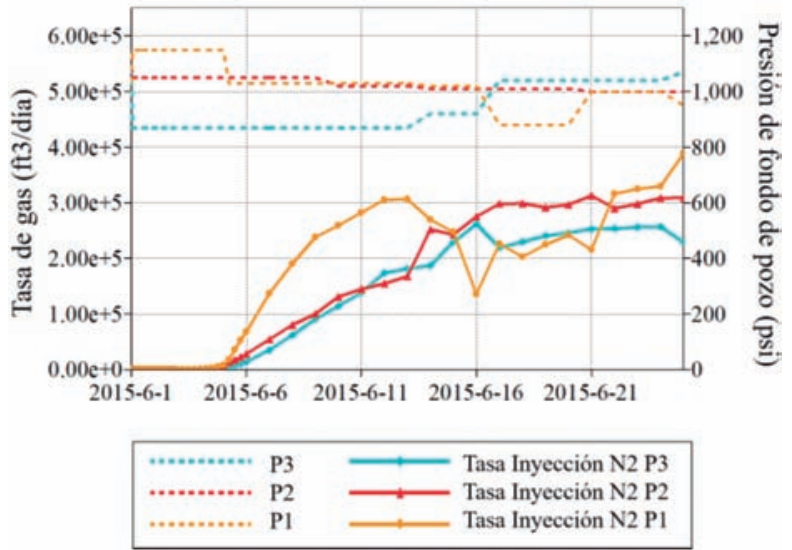

Figura 9. Pronóstico de la PCN con homogenización del frente de Nitrógeno.

\section{Implementación de la prueba de conectividad}

Con base en la etapa de diseño, y considerando los objetivos de la PCN, la implementación de la prueba se dividió en cuatro fases, de la siguiente manera:

- Fase 1: Línea base. Se definió una línea de base que incluye la tasa de producción de petróleo, agua y gas, así como sus propiedades físicas y composiciones en la estrategia de monitoreo, con el fin de identificar cualquier desviación en las propiedades extensivas e intensivas causadas por la inyección de nitrógeno.

- Fase 2: Irrupción de nitrógeno en los pozos productores de primera línea. Los esfuerzos de monitoreo se centraron en los parámetros de los pozos (variables del sistema de levantamiento artificial, presión de la cabeza y de fondo), la tasa de gas y la composición del gas en los pozos productores de primera línea para identificar adecuadamente los tiempos de irrupción del Nitrógeno.

- Fase 3: Homogenización. Se centra en la ejecución de maniobras operacionales en los pozos de producción de primera línea para ajustar la BHP y lograr tasas de producción lo más uniforme posible. Esto ayudaría a crear una ruta de gas entre el inyector y los productores que no se convierta en un canal preferencial hacia uno o dos de ellos al inyectar aire. Los parámetros que se deben ajustar incluyen la frecuencia de operación de la bomba electro sumergible (ESP) y el manejo de choques o válvulas de estrangulación en superficie.

- Fase 4: Irrupción de nitrógeno en los pozos productores de segunda línea. Similar a la Fase 2, pero en la segunda línea de productores. Esto incluye monitoreo incluso después de que la inyección de nitrógeno haya cesado.

Previo a la etapa de línea base, el pozo inyector fue perforado en el intervalo seleccionado y se realizó la operación de limpieza y preparación del pozo. La unidad criogénica para la inyección de nitrógeno se movilizó al sitio, y se instalaron los dispositivos para monitorear la composición, tasa y presión del gas producido. La duración real de cada una de las etapas contrastadas con las planificadas en el diseño se resumen en la Figura 10. 


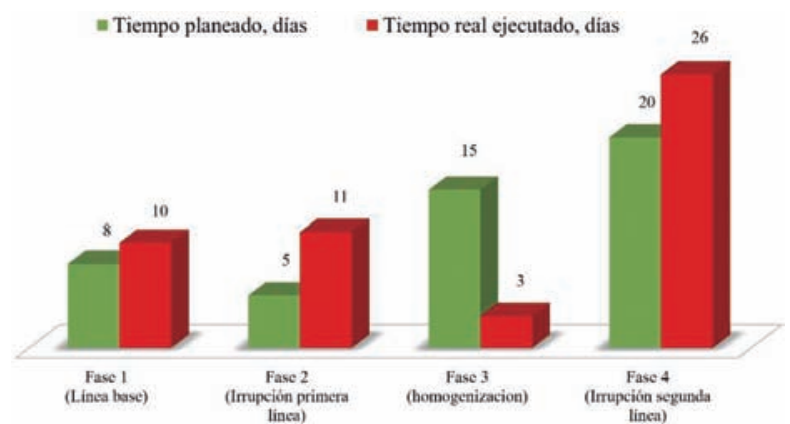

Figura 10. Duración de cada una de las fases de la PCN: Planeado Vs Ejecutado

La Fase 1 duró dos días más de lo esperado, debido a un retraso en comenzar la inyección de nitrógeno. La línea base se estableció con éxito. Las mediciones de producción mediante pruebas de separación se llevaron a cabo en cada uno de los productores de primera línea. Mediante cromatografía de gases se determinó que la fracción molar de nitrógeno en el gas de producción en promedió era de $4-5 \%$, justo antes de la inyección, que es un parámetro relevante para el balance de nitrógeno. El avance del nitrógeno ocurrió más tarde de lo previsto por la simulación para la tasa de inyección dada. Esto dio como resultado que la Fase 2 durara 11 días comparado con los 5 días estimados por la simulación numérica.

Dos de los pozos productores de primera línea (P1 y P2) evidenciaron un aumento significativo de nitrógeno en el gas producido en un periodo de 12 horas de diferencia, el día 8 después de haber iniciado la inyección. Finalmente, El nitrógeno irrumpió en el último pozo de primera línea (el pozo P3) el día 10 con mediciones de confirmación que duraron hasta el día 11.

El período de homogeneización (Fase 3) estaba programado para durar 15 días. Sin embargo, después de la irrupción del Nitrógeno, las ESP instaladas en los tres productores comenzaron a presentar un bajo rendimiento hasta que finalmente los pozos tuvieron que ser cerrados. En consecuencia, esta etapa duró solo tres días después de la fase de irrupción. Durante este tiempo, las maniobras en los controles del pozo permitieron tener una distribución de nitrógeno más estable entre los pozos productores.

La Fase 4 se llevó a cabo con los pozos productores de primera línea cerrados. Esto dio como resultado una irrupción del $\mathrm{N}_{2}$ relativamente rápido en los pozos de segunda línea (15-24 días desde el inicio de la inyección de nitrógeno). En ese momento, se detuvo la inyección de nitrógeno. Sin embargo, se continuó el monitoreo en los pozos de segunda y tercera línea, con el fin de determinar la cantidad de nitrógeno producido en cada pozo, para obtener información sobre las rutas de flujo preferenciales. En total, esta etapa duró 26 días. El nitrógeno se detectó en dos pozos más allá de la segunda línea, que se ubican en lo alto de la estructura, aunque en concentraciones muy bajas.

La tasa de inyección de nitrógeno real y de diseño se representa en la Figura 11. La tasa de inyección de diseño se modificó de la reportada en el Caso 3 de la Tabla 3 cercana a la prueba, considerando una BHP menor a la esperada en uno de los pozos productores de primera línea, que podría poner en riesgo la irrupción de Nitrógeno de manera óptima en los tres pozos de primera línea. Sin embargo, durante la prueba la tasa de inyección se incrementó antes de lo previsto con el fin de promover la irrupción. Posteriormente, se mantuvo constante ya que la irrupción en los pozos productores de segunda línea fue rápida. El volumen total inyectado fue más alto que el planificado, debido a la duración extendida de la PCN.

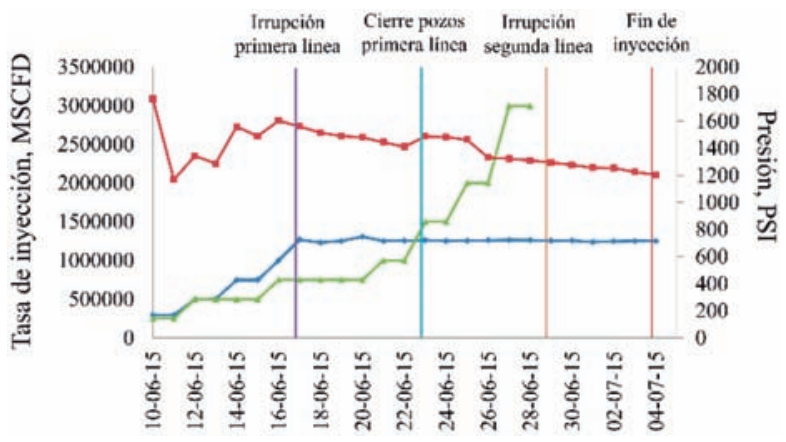

Figura 11. Tasa de Inyección y BHP medida en el pozo Inyector: diseño vs real

\section{Evaluación de la prueba de conectividad}

Los resultados obtenidos son presentados de manera separada en función de los objetivos operacionales y de yacimientos acordados para la PCN.

\section{- Resultados a nivel yacimientos}

Para determinar los tiempos de irrupción y los volúmenes de gas producidos: Las Figuras 12 y 13 resumen los tiempos de irrupción y los volúmenes acumulados de nitrógeno producido. Los tiempos de irrupción se determinaron con éxito combinando las medidas dadas por los medidores de flujo de gas y las composiciones obtenidas de los ensayos de cromatografía. Del mismo modo, se calculó la producción de nitrógeno incremental (valores superiores a la base del 4 - 5\% para la fracción molar de nitrógeno en el gas producido). Se observó 
que el gas fluía en baches discontinuos en algunos de los pozos, lo que es fuente de incertidumbre en los volúmenes totales informados.

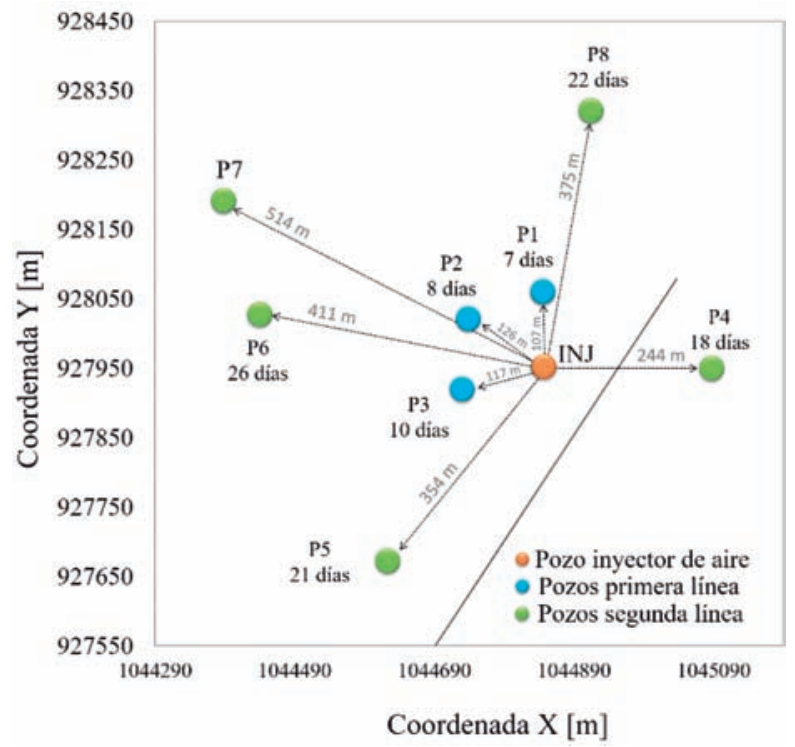

Figura 12. Mapa mostrando los tiempos de irrupción del Nitrógeno en pozos productores.

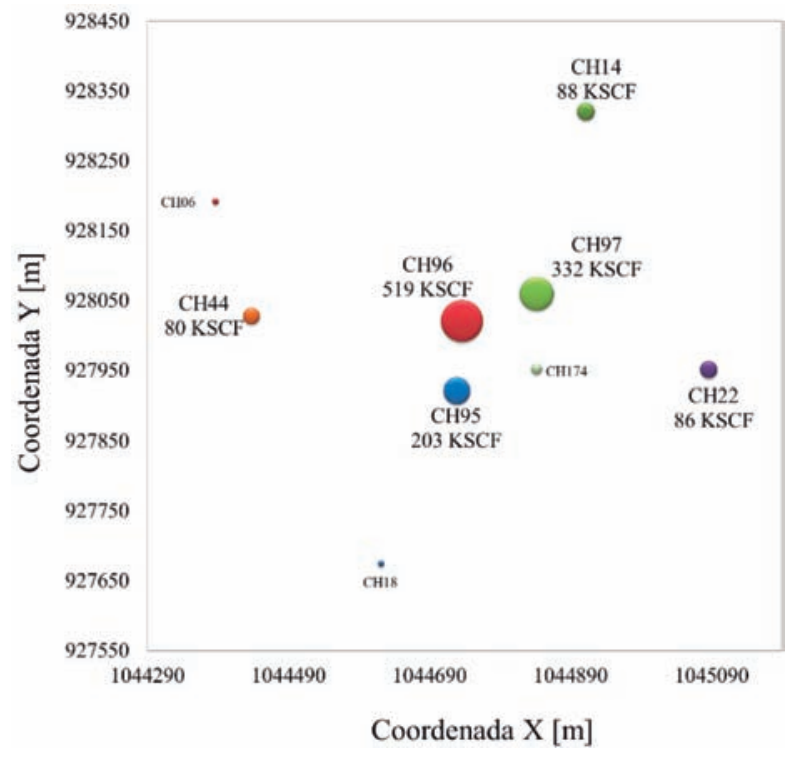

Figura 13. Mapa mostrando nitrógeno producido acumulado.

Para verificar la conectividad: Inicialmente se verificó la conectividad entre el inyector y los pozos productores de primera línea. El nitrógeno irrumpió a los tres pozos con tres días de diferencia. La secuencia de irrupción fue la esperada, considerando las medidas de presión, las propiedades de la roca y la elevación de los intervalos perforados. Los tiempos de irrupción fueron más largos de lo esperado, lo que implica que el desplazamiento inmiscible es más eficiente de lo que inicialmente se suponía, por lo que los parámetros de permeabilidad relativa en los modelos de simulación deben ajustarse para reproducir esta conducta. Mientras que los pozos productores de primera línea estuvieron abiertos, no hubo detección de nitrógeno incremental en ningún otro pozo, lo que evidencia que el fluido inyectado estará mayormente contenido dentro del área piloto.

Para identificar las características geológicas: la propagación uniforme del frente de nitrógeno no sugiere evidencias de canales de flujo preferenciales o barreras de flujo en una dirección particular. Se observó nitrógeno incremental en pozos ubicados al norte (P8, $375 \mathrm{~m}, 22$ días), al sur (P5, $354 \mathrm{~m}, 21$ días), al oeste (P6, $411 \mathrm{~m}$, 26 días) y al este (P4, $244 \mathrm{~m}, 18$ días) del pozo inyector. A partir de las distancias y tiempos anteriores, se puede calcular una velocidad promedio, concluyendo que el nitrógeno fluye aproximadamente a la misma velocidad en todas las direcciones, incluido el pozo P4, aunque se identificó una falla entre el inyector y este pozo, evidenciado por un salto estructural de 140 pies, como se muestra en la Figura 14. La existencia o el carácter de esta falla deben revisarse a la luz de los resultados de la PCN. Una hipótesis factible es que la falla comunica la unidad T2_40 en el bloque del inyector con el bloque T2_60 en el bloque P4 sin restricción de flujo. Esta y otras hipótesis se evaluarán usando la simulación de yacimientos.

Adicionalmente, se evidenció la influencia de la inyección de nitrógeno en las tasas de producción en el pozo P4. Este pozo está abierto a producción en otra arena (K1) que contiene aceite de $15^{\circ}$ API. Durante la PCN y como se observa en la Figura 15, la gravedad API producida cayó a un promedio de 10. Esto se explica por el mejor soporte de presión en la arena T2 (que produce petróleo con gravedad API entre 8 y 9) y la expansión de gas tiempo después de que la inyección de nitrógeno había concluido. Otra diferencia entre las arenas T2 y $\mathrm{K} 1$ es el corte de agua y la salinidad. Ambos parámetros siguieron tendencias similares a la de la gravedad API, dominando los valores representativos de arena T2 durante y después de la PCN.

Las tendencias en el volumen total de gas producido (Figura 13) confirman la uniformidad de la distribución de nitrógeno alrededor del pozo del inyector en el área piloto. El pozo más cercano que no exhibió ningún nitrógeno incremental fue el P7. Este comportamiento se explica por el hecho de que el pozo cercano P6 drena el gas libre transportado a esta vecindad. El balance de gas total (inyección versus producción) se presenta en la Figura 16. 


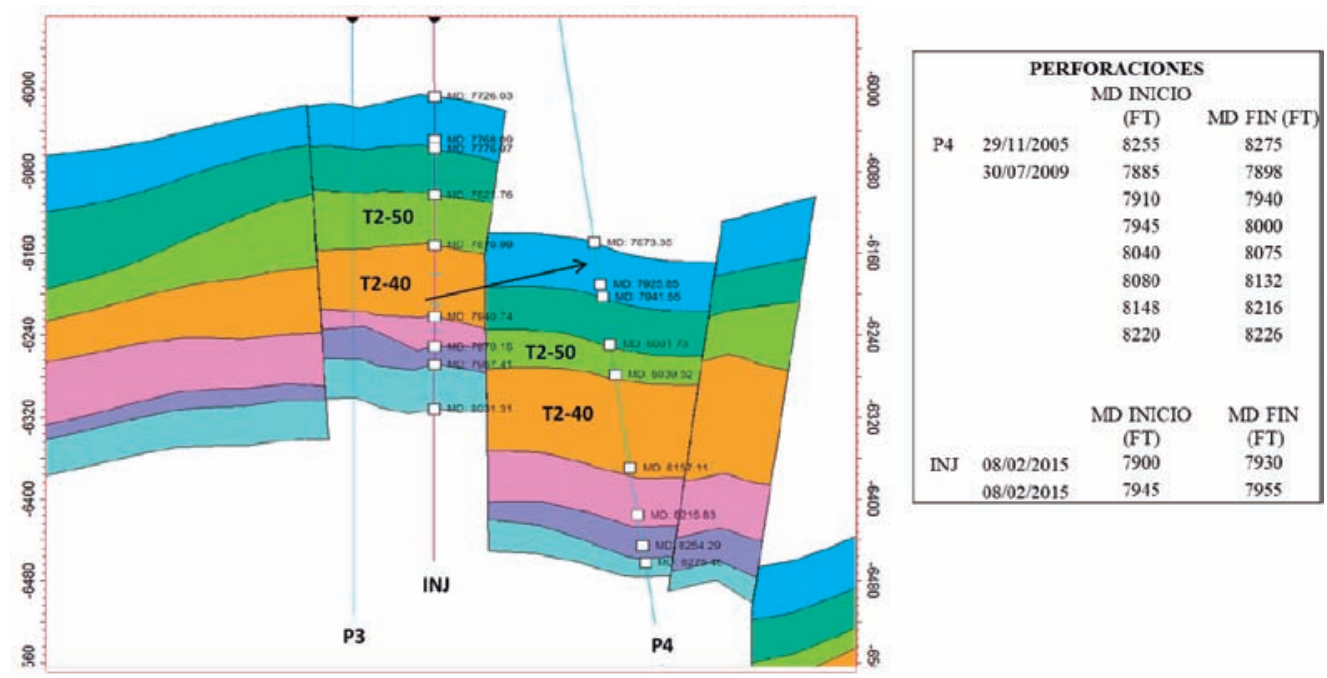

Figura 14. Falla entre el pozo Inyector y el P4

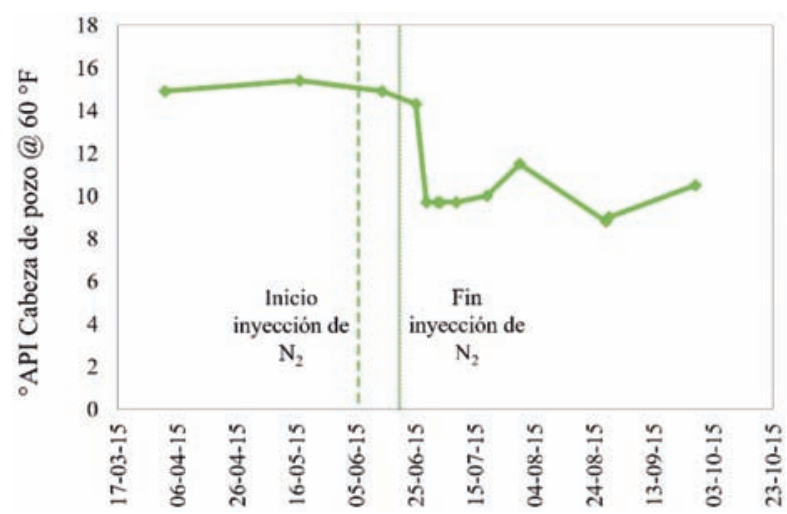

Figura 15. Variación de la gravedad API del aceite producido en el pozo $\mathrm{P} 4$.

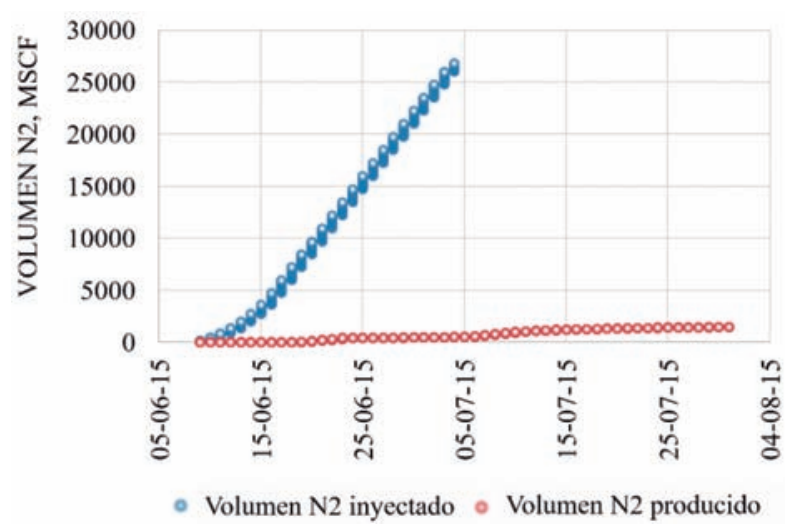

Figura 16. Volumen de Nitrógeno inyectado y producido acumulado.

A partir del balance de $\mathrm{N}_{2}$ inyectado y producido, se estimó que solo se produjo alrededor del 5,5\% del nitrógeno inyectado. Este volumen pudiese haber sido mucho mayor si los pozos productores de primera línea no hubieran sido cerrados. La mayor parte de este volumen de gas restante se distribuiría en zonas de baja saturación, que, no obstante, constituyen rutas de flujo preferentes para la inyección de aire. Esta es una característica positiva, teniendo en cuenta que el aire inyectado debe desplazarse efectivamente lejos del inyector para obtener una ignición segura. Teniendo en cuenta que estas vías de flujo crecen uniformemente alrededor del pozo inyector, es favorable para el desarrollo homogéneo del frente de combustión. La distribución geométrica de esta zona de gas puede determinarse aproximadamente mediante simulación numérica, y se debe investigar su dinámica de flujo antes de la inyección de aire.

Después de realizado el balance de gas hidrocarburo producido, se observó un exceso con respecto a los valores reportados durante la línea de base. La Figura 17 es un ejemplo de este comportamiento. La relación gas-petróleo (GOR) de referencia oscilaba entre 30 y $50 \mathrm{SCF} / \mathrm{STB}$. Tras la prueba, el GOR medido en el separador de prueba fue $420 \mathrm{SCF} / \mathrm{STB}$. Restando el nitrógeno producido, el GOR de hidrocarburo permanece cerca de 300 SCF / STB. Una estimación analítica de la vaporización de hidrocarburos en la corriente de gas debido a múltiples contactos con nitrógeno muestra que este fenómeno no puede explicar más de $50 \mathrm{SCF}$ / STB. En el balance, aproximadamente $200 \mathrm{SCF}$ / STB deben atribuirse al gas de hidrocarburo libre desplazado por el nitrógeno inyectado en el caso de este pozo (P3). Para cada productor influenciado en la PCN, se observó este exceso de volumen de gas de hidrocarburo. Los estudios de PVT de fluidos sugieren que la presión del punto de burbujeo de este crudo debe ser de aproximadamente 1000 psia, por lo que en las condiciones de la PCN, el 
yacimiento es subsaturado. Sin embargo, se han hecho observaciones de alto GOR desde el principio de la vida del campo, cuando la presión era superior a 3.000 psia. La presencia de gas libre no debería representar una amenaza significativa para el proceso de combustión in situ, pero si debe modelarse y ajustarse para obtener predicciones fiables del rendimiento del proceso.

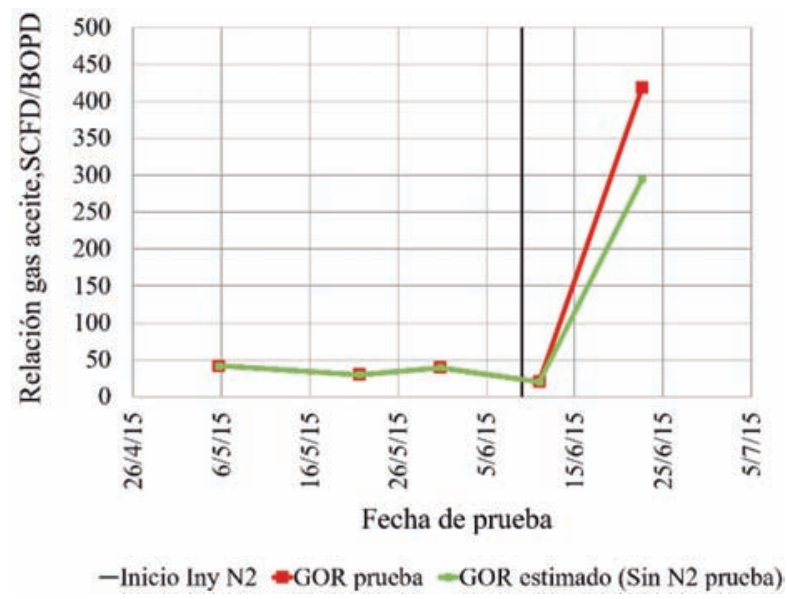

Figura 17. GOR medido en el pozo P3.

- Resultados a nivel operacional

Para validar inyectividad y completamiento del pozo inyector: El completamiento del pozo presenta materiales resistentes a alta temperatura, alta presión y corrosión (cemento térmico, aleación de cromo, grasa inerte de oxígeno en uniones, empaques térmicos) que no se han probado dado que la inyección de aire no ha iniciado y la temperatura permanece en el valor original del yacimiento. En ese orden, cada una de las juntas que componen el completamiento del pozo inyector se probaron individualmente llenando con agua y presurizando con nitrógeno, evidenciando una contención de presión efectiva.

Se realizó una prueba de inyectividad de agua, que consta de ocho períodos de tiempo (flujo / cierre), el último de los cuales fue un lapso de tiempo de permanencia de 240 horas. El potencial de inyección se estimó por encima de $3 \mathrm{E}+06 \mathrm{SCF} /$ día durante todo el intervalo perforado de 40 pies.

Para validar el desempeño del sistema de levantamiento en pozos productores: La viscosidad del aceite esperada en condiciones de producción en frío $\left(150-180^{\circ} \mathrm{F}\right)$ es del orden de miles de centipoises. Se anticipa que los niveles dinámicos de fluido estarán por debajo de 6000 pies de profundidad (TVD). Para estas condiciones, las bombas electro sumergibles (ESP) deben instalarse por debajo del nivel de 7000 pies. Las ESP's normalmente operaban en condiciones de producción primaria. Con una mayor producción de gas como producto de la inyección de nitrógeno, que se libera intermitentemente, según lo detectado por los medidores de flujo de gas instalados, las bombas estaban sujetas a bloqueos debido a que el gas entraba directamente en la succión. La solución anticipada a esta falla fue el diseño de un aumento gradual en la tasa de inyección de gas, pero manteniendo el nivel del fluido a una distancia segura de la succión de la bomba. En la ejecución de la prueba esto no fue suficiente, debido a que el gas se producía en baches, incrementaba considerablemente las relaciones gas /líquido y por consiguiente se bloqueó la bomba. Esto implica que el sistema de levantamiento artificial requiere un rediseño para operar adecuadamente durante la etapa de inyección de aire.

Para evaluar las maniobras en los pozos productores durante la etapa de homogenización: dada la corta duración de esta etapa de homogenización y el rendimiento del ESP después de la irrupción del Nitrógeno, este objetivo se cumplió parcialmente. Sin embargo, se logró un cierto nivel de control de los choques de los pozos y la frecuencia de operación de la bomba. La Figura 18 muestra la sensibilidad de la presión del pozo en el fondo del pozo (BHP) en función de la frecuencia de la bomba.

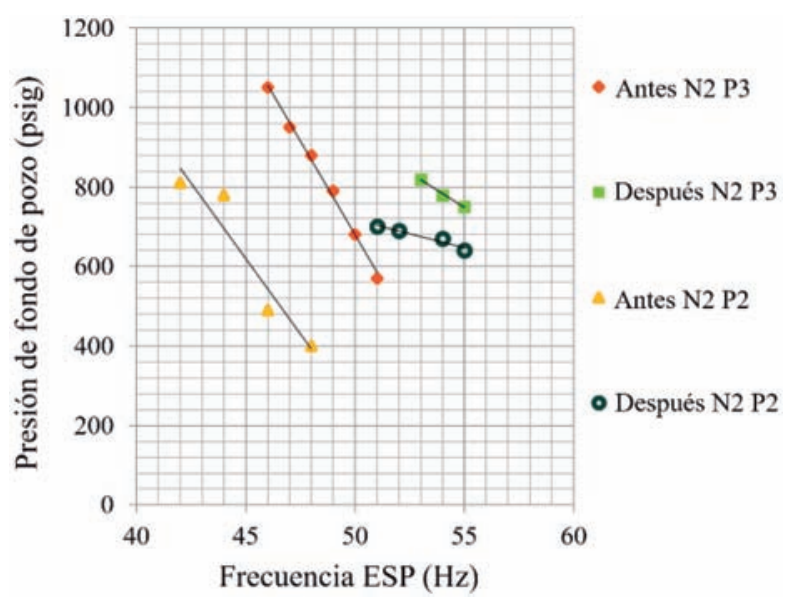

Figura 18. Presión de fondo medida en el pozo P3.

Antes de la inyección de nitrógeno, la BHP tenía un rango de variación más amplio que el observado durante la inyección de nitrógeno, para ambos pozos reportados. Este efecto es doble: por un lado, limita la maniobrabilidad para promover una perturbación en el campo de presión que permite la redirección del flujo de nitrógeno, pero, por otro lado, evidencia un índice de productividad del pozo más alto, como resultado del 
soporte de presión que el inyector proporciona. Esto está sustentado por el incremento en la producción de fluidos en los pozos de primera línea observado muy puntualmente durante la PCN.

Para validar la estrategia de monitoreo y toma de datos en el campo: Aunque las instalaciones y equipos utilizados para monitorear y medir la tasa y composición del gas fueron temporales y se dispondrá de un esquema diferente para la operación del piloto, se evidenció que las frecuencias y los métodos fueron apropiados para detectar los tiempos de irrupción del nitrógeno y hacer buenas estimaciones para el balance de masa. Algunas lecciones de aprendizaje se transferirán a la etapa de operación piloto.

\section{Conclusiones}

- Los tiempos de avance del nitrógeno en los pozos productores de primera y segunda línea evidenciaron una progresión uniforme del frente de gas en todas las direcciones.

- La conexión entre el pozo inyector y el pozo productor $\mathrm{P} 4$ es más directa de lo previsto, solicitando una reevaluación del modelo geológico del área piloto ara el área piloto.

- Se observó gas libre de hidrocarburo durante la prueba en todos los pozos donde se detectó nitrógeno. Esto implica que el modelo de fluidos debe revisarse para obtener mejores predicciones.

- Hubo evidencia de una mejor productividad de los pozos de primera línea debido a la inyección de nitrógeno en las arenas $\mathrm{T} 2$.

- El potencial de inyección de aire se estimó con base a una prueba de inyectividad de agua por encima de $3 \mathrm{E}+06 \mathrm{SCF} /$ día de gas en todo el intervalo perforado de 40 pies.

- El flujo intermitente de gas causó la falla de la ESP por bloqueo de gas en los tres pozos productores de primera línea. Se debe formular un diseño más robusto del sistema de levantamiento, considerando flujo de gas en baches.

- El cierre de los pozos de primera línea impidió realizar maniobras para homogeneizar la progresión del gas con tiempo suficiente para observar las respuestas estabilizadas. Sin embargo, se espera que bajo un mejor funcionamiento de los sistemas de levantamiento, la homogenización del frente de combustión sea posible.

- Los resultados de la PCN indican que la implementación del proceso de inyección de aire en campo podría ser exitosa y ayudarán a mejorar el desempeño del proceso, si se llevan a cabo las siguientes acciones:
- Modificación de los sistemas de levantamiento para manejo de mayores relaciones gas/líquido.

- Definir las condiciones de operación del pozo P4 durante el proceso de inyección de aire.

- Optimizar la estrategia de monitoreo de acuerdo con el comportamiento del flujo del gas presentado durante la $\mathrm{PCN}$.

- Actualización del modelo de simulación numérica.

\section{Nomenclaturas}

$\mathrm{BHP}=$ Presión de fondo de pozo (Bottom Hole Pressure)

$\mathrm{STB}=$ Barriles en tanque (Stock Tank Barrels)

OIIP $=$ aceite original en sitio (Original Oil in Place)

$\mathrm{SCF}=$ pies cúbicos estándar (Standard Cubic Feet)

GOR $=$ relación Gas-aceite (Gas-Oil Ratio)

\section{Agradecimientos}

Los autores agradecen a los siguientes consultores quienes proveyeron sus puntos de vista para el desarrollo de la PCN: Louis Castanier, Claude Gadelle y Alex Turta. Adicionalmente, a Jorge Mario Padilla y Jorge Mario Palma por el Soporte en las actividades de simulación numérica. De igual manera, a Fernan Isdwar Rojas, Andrés Felipe Trujillo, José Luis Gualdrón, Alcibiades Sosa Rozo, Pablo Emilio Solarte, Jairo Narváez, Fabio Acosta y William Jiménez, por su gestión y esfuerzos durante la operación en campo. Finalmente, a Ecopetrol por permitir publicar estos resultados.

\section{Referencias}

1. Aya, C. L. D., \& Trujillo, M. L. (2013). La inyección de aire como proceso de recobro mejorado en Colombia: Selección y evaluación técnica. Fuentes: El reventón energético, 11(2), 3.

2. Bottia-Ramirez, H., Aguillon-Macea, M., LizcanoRubio, H., Delgadillo-Aya, C. L., \& Gadelle, C. (2017). Numerical modeling on in-situ combustion process in the Chichimene field: Ignition stage. Journal of Petroleum Science and Engineering, 154, 462-468.

3. Burger, J. G. (1972). Chemical aspects of in-situ combustion-heat of combustion and kinetics. Society of Petroleum Engineers Journal, 12(05), 410-422.

4. Castañeda, A. M., Ruiz, M. C. \& Navarro, S. F. (2014). Modelado de procesos de combustión in situ. Fuentes: El reventón energético, 12(1), 5. 
5. Fassihi, M. R.; Brigham, W.; Ramey. Reaction Kinetics of in-situ combustion: Part 1-Observations. Paper SPE 8907. Agosto, 1984.

6. Glandt, C. A., Pieterson, R., Dombrowski, A., \& Balzarini, M. A. (1999, January). Coral creek field study: a comprehensive assessment of the potential of high-pressure air injection in a mature waterflood project. In SPE midcontinent operations symposium. Society of Petroleum Engineers.

7. Hutchinson, H. L., Spivak, A., \& Johnson, L. A. (1979, January). Simulation Study Of The LETC TS2C In Situ Combustion Test In Utah Tar Sands. In SPE Annual Technical Conference and Exhibition. Society of Petroleum Engineers.

8. Johnson Jr, L. A., Fahy, L. J., Romanowski, L. J., Barbour, R. V., \& Thomas, K. P. (1980). An echoing in-situ combustion oil recovery project in a Utah tar sand. Journal of Petroleum Technology, 32(02), 295-305.

9. Moore, R.G. Mehta, S.A. and Ursenbach, M.G. A Guide to High-Pressure Air Injection (HPAI) Based Oil Recovery. Paper SPE 75207, presented at the SPE/DOE Improved Oil Recovery Symposium, Tulsa, OK, U.S.A., April 2002.
10. Navarro, W., Muñoz, S. F., Ordoñez, A. \& Bottia, $H$ (2016). Ajuste de una prueba de tubo de combustión usando un modelo simplificado de reacciones. Revista Fuentes, 14(2), 99-109.

11. Pebdani, F. N., Longoria, R., Wilkerson, D. N., \& Venkatesan, V. N. (1988, January). Enhanced Oil Recovery by Wet In-Situ Oxygen Combustion: Esperson Dome Field, Liberty County, Texas. In SPE Annual Technical Conference and Exhibition. Society of Petroleum Engineers.

12. Reyes, J. P., Trujillo, M. L., Velásquez, E. N., \& Aya, C. L. D. (2017). Metodología para la selección y priorización de yacimientos de crudo liviano candidatos a la inyección de aire: caso de aplicación. Fuentes: El reventón energético, 15(1), 41-48.

13. Sarathi, P. S. In-situ combustion handbook-principles and practices. Final Report, November 1998. Performed Under Contract No. DE-AC2294PC91008.

14. Turta, A. T., \& Singhal, A. K. (2001). Reservoir engineering aspects of light-oil recovery by air injection. SPE Reservoir Evaluation \& Engineering, 4(04), 336-344.

Recepción: 26 septiembre de 2018

Aceptación: 21 de noviembre de 2018 